\title{
A Model of Facial Expression for 3D Avatar
}

\author{
Zhen Liu \\ Faculty of Information Science and Technology, Ningbo University, \\ 315211, China \\ liuzhen@nbu.edu.cn
}

\begin{abstract}
Modeling 3D avatar is very useful in e-learning software, a believable 3D avatar can have complex facial expression, and how to construct complex facial expression is a very interesting topic. The concept of emotion vector is presented. Emotion state of a 3D avatar can be transformed to an emotion vector that is calculated by basic emotion vector. The concept of expression vector is established, expression can be transformed to expression vector that is calculated by basic expression vector. Emotion mapping is set up from emotion vector space to expression vector space. A preliminary experiment is carried on microcomputer.
\end{abstract}

Keywords: emotion, emotion vector, emotion synthesis.

\section{Introduction}

Modeling 3D avatar is very useful in e-learning software, a believable 3D avatar can have complex facial expression, and how to construct complex facial expression is a very interesting topic.

Parke first presented the research paper on facial animation [1], and there are many methods of facial animation in now days. The prior research mainly concentrated on driving some local movements of face effectively, and little on whole movement of face. For example, the physical model is an effective method of controlling muscle's movement on face, the relation between muscle movement and facial expression's semantic information is not clear. Some methods emphasized on synthesis of facial expression by local movement, Ekman first proposed the famous FACS system that can describe facial expression by Action Units [1], but the method has many limitations for synthesis of complex expression. Noh et al. presented an ingenious technique to clone expression vector from one character to another, the purpose utilizes existing expression information, but it cannot create new expression [2]. However, the interpolation technique of facial expression can utilize whole information of the face effectively [3], it only produces little new expression and lacks the quantitative description of the emotion meaning.

A synthesis model of emotion is presented on basic emotion in this paper, the model attempts to set up a formal description of complex emotion according to each intensity of basic emotion. 


\section{A Computational Model of Emotion Synthesis}

For a certain $3 \mathrm{D}$ avatar, $\boldsymbol{B} \boldsymbol{E}$ is a basic emotion class set, $\boldsymbol{B} \boldsymbol{E}=\left\{\right.$ be $_{1}, \ldots \ldots$, be $\left._{\mathrm{N}}\right\}, i \in[1$, $\mathrm{N}]$, be $_{i}$ is a basic emotion (such as happiness). $\mathrm{N}$ is the number of basic emotion class. $E I_{i}(t)$ is the intensity of $\mathrm{be}_{i} . E I_{i}(t) \in[0,1], t$ is time variable. $\boldsymbol{b} \boldsymbol{e}_{i}$ is the unit vector of be $_{i .}$. For example, $\boldsymbol{b} \boldsymbol{e}_{l}=\{1, \ldots, 0\}, \boldsymbol{b} \boldsymbol{e}_{N}=\{0, \ldots, 1\}$. Let $\mathrm{E}$ is emotion state, $\boldsymbol{E}$ is represented emotion vector of E, the projection length of $\boldsymbol{E}$ on $\boldsymbol{b} \boldsymbol{e}_{i}$ is $E I_{i}(t) . \boldsymbol{E}$ can be represented as formula (1):

$$
\boldsymbol{E}=\sum_{i=1}^{N} E I_{i}(t) \boldsymbol{b} \boldsymbol{e}_{i}
$$

Let $\boldsymbol{E}_{1}$ and $\boldsymbol{E}_{2}$ are two emotion vectors, the synthesis of $\boldsymbol{E}_{l}$ and $\boldsymbol{E}_{2}$ is represented as $\boldsymbol{E}_{l}$ $+\boldsymbol{E}_{2}$. There is a formula (2).

$$
\boldsymbol{E}_{l}+\boldsymbol{E}_{2}=\sum_{i=1}^{N}\left[E I_{i 1}(t) \boldsymbol{b} \boldsymbol{e}_{i 1}+E I_{i 2}(t) \boldsymbol{b} \boldsymbol{e}_{i 2}\right]
$$

Let $\boldsymbol{E P}$ is the set of all emotion vectors, if any element of $\boldsymbol{E P}$ satisfies to formula (1)(2), $\boldsymbol{E P}$ is called emotion vector space, $\boldsymbol{b} \boldsymbol{e}_{i}$ is called the basic emotion vector.

A face's geometry model is described by polygons, the location of any vertex can be represented as vector $\boldsymbol{v}_{k}, k \in[0, \mathrm{~L}], \mathrm{L}$ is the number of all vertex. Let $\boldsymbol{V}$ is a vector for all vertex, $\boldsymbol{V}$ is called expression vector. $\boldsymbol{V}$ is represented as formula (3).

$$
\boldsymbol{V}=\left(\boldsymbol{v}_{1}, \ldots \ldots, \boldsymbol{v}_{L}\right) \text {. }
$$

In general, there are some expression vector, $p n$ is the number of expression vector, $\boldsymbol{V}_{i}$ is an expression vector, $i \in[0, p n], S Y$ is an synthesis function among all $\boldsymbol{V}_{i}, \boldsymbol{F} \boldsymbol{V}$ is calculated by formula (4):

$\boldsymbol{F} \boldsymbol{V}=S Y\left(\boldsymbol{V}_{1}, \ldots \ldots, \boldsymbol{V}_{p n}\right)$.

In general, $\boldsymbol{E P}$ is emotion vector space, $\boldsymbol{F} \boldsymbol{V}$ is called expression vector space, $T$ is a emotion function from $\boldsymbol{E P}$ to $\boldsymbol{F} \boldsymbol{V}$, for any $\boldsymbol{E} \in \boldsymbol{E P}, T(\boldsymbol{E}) \in \boldsymbol{F} \boldsymbol{V}$. Let $\boldsymbol{b} \boldsymbol{e}_{i}$ is a unit vector, $i \in[1, \mathrm{~N}], T\left(\boldsymbol{b}_{i}\right)$ is called base expression vector. If $p n=\mathrm{N}, \boldsymbol{F} \boldsymbol{V}$ is calculated by formula (5):

$$
\boldsymbol{F} \boldsymbol{V}=S Y\left(T\left(\boldsymbol{b} \boldsymbol{e}_{1}\right)_{1}, \ldots \ldots, T\left(\boldsymbol{b} \boldsymbol{e}_{N}\right)\right)
$$

In order to simplify the formula (5), let $S Y$ is linear function, $\lambda_{i}$ is the corresponding interpolation function of $\boldsymbol{V}_{i}$, the sum of all $\lambda_{i}$ is equal to 1 , let $\lambda_{i}=E I_{i}, \boldsymbol{F} \boldsymbol{V}$ is calculated by formula (6):

$$
\boldsymbol{F} \boldsymbol{V}=\sum_{i=1}^{N}\left(E I_{i}\right) T\left(\boldsymbol{b} \boldsymbol{e}_{i}\right) .
$$




\section{A Demo of Complex Facial Expression}

A demo of synthesis on expression by formula (6) is realized on pc, the programming tools are Visual c++ language and Direct3D API. In the demo, only six basic facial expressions are selected. Six basic facial expressions are shown in Fig.1, some of synthesis results are shown in Fig. 2. For example, in Fig. 2(1), "1/2 happiness $+1 / 2$ sadness" is represented the synthesis of happiness and sadness, each emotion intensify is equal to $1 / 2$.
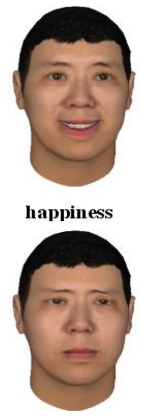

sadness
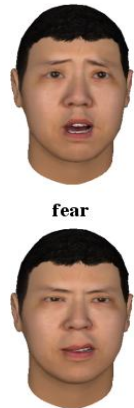

disgust

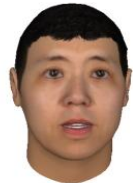

surprise

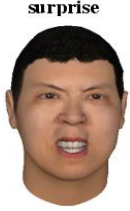

anger

Fig. 1. Six basic facial expressions

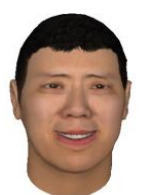

(1)

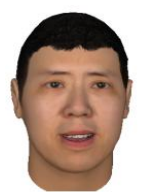

(4)

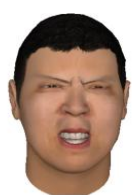

(2)

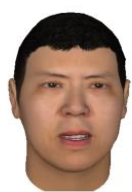

(5)

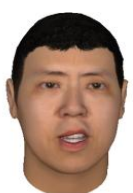

(3)

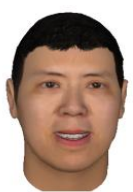

(6)

Fig. 2. (1)1/2happiness+1/2sadness;(2)1/2sadness+1/2anger;(3)1/2surprise+1/2disgust;(4)1/3happiness $+1 / 3$ fear+1/3disgust;(5)1/3sadness+1/3disust+1/3anger;(6)1/3happiness+1/3sadness $+1 / 3$ anger

\section{Conclusion and Future Work}

A model of facial expression for 3D avatar is presented in this paper. The paper improves the interpolation technique of facial expression. The concept of emotion vector space is introduced, and a basic emotion can be regarded as a unit vector in emotion vector space. The concept of expression vector space is also introduced, and an expression vector can describe a facial expression. An emotion function can transform a basic emotion to a base vector in expression vector space. In order to simplify an 
emotion function, an expression vector can be described by linear combination of base vector. A preliminary experiment indicates that basic emotion can produce many new emotions.

\section{Acknowledgements}

The work described in this paper was co-supported by science and technology project of Zhejiang Province Science Department (grant no: 2006C33046), forepart professional research of ministry of science and technology of the People's Republic of China (grant no: 2005cca04400), University Research Project of Zhejiang Province Education Department (grant no: 20051731).

\section{References}

1. Parke, F.I., Waters,K.: computer facial animation, Wellesley, Boston, USA: AK Perters (1996)

2. Noh, Jun-yong, and Ulrich Neumann. Expression Cloning, In Proceedings of ACM SIGGRAPH2001 Conference (2001) 277-288

3. Blanz, V., Vetter, T.: A Morphable Model for the Synthesis of 3D Faces, In Proceedings of ACM SIGGRAPH 99 Conference (1999) 187-194 